\title{
Treatment of Dual Genotype Chronic Hepatitis C: Case Series
}

\author{
Lokesh K. Jha ${ }^{1} \cdot$ Jorge Gilbert $^{1}$
}

Received: 16 March 2016/Accepted: 28 March 2016/Published online: 5 April 2016

(C) Springer Science+Business Media New York 2016

\section{Dear Editor,}

There has been a lot of progress in the treatment of chronic hepatitis $\mathrm{C}$ in the last 2 years, but so far, there is no study on treatment of dual genotype chronic hepatitis $\mathrm{C}$ as dual genotypes are rare [1]. It is also challenging to get treatment medication approved by insurance company for these kinds of patients.

We have so far treated three patients with chronic hepatitis $\mathrm{C}$ dual genotype.

1. A 60-year-old male with liver cirrhosis (confirmed with liver biopsy) secondary to chronic hepatitis C genotypes 1a and 2, treatment naïve: He was treated with 16-week course of sofosbuvir/ledipasvir and ribavirin $600 \mathrm{mg}$ BID. He did not have any side effects due to medication. He did have some issues with interruption of treatment while waiting for refill on his prescription after he completed 12 weeks of treatment. His HCV RNA before starting treatment was 2143667. HCV RNA was undetectable at 4 and 12 weeks. He was able to achieve SVR 12.

2. A 73-year-old female with chronic hepatitis C, genotypes $1 \mathrm{~b}$ and 3 with stage 2 fibrosis on liver biopsy: She previously had genotype 1 and had relapse when she was treated with interferon and ribavirin, and also developed interferon-associated Graves' disease. Eight years later when directly acting antivirals became available, repeat hepatitis $\mathrm{C}$ genotype showed that she

Lokesh K. Jha

drljha@gmail.com

1 Sanford Center for Digestive Health, Sanford School of Medicine, University of South Dakota, Sioux Falls, SD, USA has genotypes $1 \mathrm{~b}$ and 3 . She did not have any risk factors for hepatitis $\mathrm{C}$ reinfection after prior treatment. She was treated with sofosbuvir $400 \mathrm{mg}$ daily (total duration 24 weeks), simeprevir $150 \mathrm{mg}$ daily (total duration 12 weeks), and Ribavirin 400 mg BID (total duration 24 weeks). She tolerated the medication without any side effects, and there was no interruption in the treatment. Her HCV RNA before starting the treatment was 4760000 . HCV RNA was $<15$ at 4 weeks, undetectable at 6 and 12 weeks. She was able to achieve SVR 12.

3. A 55-year-old male with liver cirrhosis (seen in CT scan) secondary to chronic hepatitis $\mathrm{C}$, genotypes $1 \mathrm{a}$ and 2, treatment naïve: He was treated with 16-week course of sofosbuvir/ledipasvir and ribavirin $600 \mathrm{mg}$ BID. He tolerated the medication without any side effects, and there was no interruption in the treatment. His HCV RNA before starting treatment was 6460000 . HCV RNA was undetectable at 4 and 12 weeks of treatment. He was able to achieve SVR 12.

\section{Conclusion}

Although dual genotypes of chronic hepatitis $\mathrm{C}$ are rare, these patients can also be successfully treated with newer directly acting antiviral agents.

\section{Reference}

1. AASLD/IDSA HCV Guidance: recommendations for testing, managing, and treating hepatitis C. http://www.hcvguidelines. org/. 